\title{
Associations between Tactile Sensory Threshold and Postural Performance and Effects of Healthy Aging and Subthreshold Vibrotactile Stimulation on Postural Outcomes in a Simple Dual Task
}

\author{
Marius Dettmer, ${ }^{1,2}$ Amir Pourmoghaddam, ${ }^{1,2}$ Beom-Chan Lee, ${ }^{2}$ and Charles S. Layne ${ }^{2}$ \\ ${ }^{1}$ Memorial Bone \& Joint Research Foundation, 1140 Business Center Drive, Suite 101, Houston, TX 77043-2740, USA \\ ${ }^{2}$ Center for Neuromotor and Biomechanics Research, John P. McGovern Campus, 2450 Holcombe Boulevard, Houston, \\ TX 77021-2040, USA
}

Correspondence should be addressed to Marius Dettmer; marius.dettmer@uth.tmc.edu

Received 28 October 2015; Accepted 22 March 2016

Academic Editor: Abebaw Yohannes

Copyright (c) 2016 Marius Dettmer et al. This is an open access article distributed under the Creative Commons Attribution License, which permits unrestricted use, distribution, and reproduction in any medium, provided the original work is properly cited.

\begin{abstract}
Specific activities that require concurrent processing of postural and cognitive tasks may increase the risk for falls in older adults. We investigated whether peripheral receptor sensitivity was associated with postural performance in a dual-task and whether an intervention in form of subthreshold vibration could affect performance. Ten younger (age: 20-35 years) and ten older adults (70-85 years) performed repeated auditory-verbal 1-back tasks while standing quietly on a force platform. Foot sole vibration was randomly added during several trials. Several postural control and performance measures were assessed and statistically analyzed (significance set to $\alpha$-levels of .05). There were moderate correlations between peripheral sensitivity and several postural performance and control measures ( $r=.45$ to .59$)$. Several postural performance measures differed significantly between older and younger adults $(p<0.05)$; addition of vibration did not affect outcome measures. Aging affects healthy older adults' performance in dual-tasks, and peripheral sensitivity may be a contributor to the observed differences. A vibration intervention may only be useful when there are more severe impairments of the sensorimotor system. Hence, future research regarding the efficacy of sensorimotor interventions in the form of vibrotactile stimulation should focus on older adults whose balance is significantly affected.
\end{abstract}

\section{Introduction}

Aging is known to cause multiple changes in anatomy and physiology of the human body. One significant modification is observed in sensory systems that provide information about body configurations and properties of the external environment. In general, there is a constant decline of sensory functioning and associated sensitivity to stimuli, which begins around the 4th to 5th decade of life with a more rapid decline during the 7th decade. This decline, in addition to loss of cognitive/executive function, leads to problems in sensorimotor processing [1]. Aging of the sensorimotor systems involved in assuring postural stability is a main contributor to the increased prevalence of balance impairments and associated falls in older adults [2-4]. In 2007, there were approximately 1.5 million falls of older citizens (75 years of age and older) reported in the US and approximately 400.000 patients required hospitalization after falls [5]. It is believed that progressive decline of sensory systems function (e.g., plantar mechanoreceptors, vestibular system, muscle spindle afferents, vision) and impairments in proprioceptive-spinal circuits lead to issues regarding the detection of small fluctuations in postural orientation during upright stance [6], which in turn increases the risk for postural balance performance decline and falls [7].

Such deterioration of function often interacts with the affordances posed by specific tasks: Among the major challenges to postural stability is the simultaneous processing 
of both motor and cognitive tasks. Concurrent postural and cognitive tasking, as often experienced in activities of daily living, may pose a major challenge to older adults, who often exhibit less cognitive capacity (meaning there are fewer available cognitive resources that can be allocated to either of the two tasks) and exhibit generally lower sensorimotor and postural performance. The deterioration in either the cognitive or postural compartment (or both) of such dual-tasks can be interpreted as changes of task processing strategies or prioritization modification.

The modalities of dual-task processing in older adults have been the focus of numerous research endeavors, with major aims being the investigation of executive mechanisms, like concurrent processing modalities, prioritization, and altered behavior patterns due to aging or pathologies [3, 814]. Postural control may require cognitive resource itself, and it becomes more difficult at older age and is cognitively more demanding for older people than for younger people [15]. Empirical evidence suggests that aging has a significant effect on processing of attention mechanisms and attention capacity, which is reflected in experimental results showing differences between younger and older participants $[6,14$, $16,17]$.

Mainly, aging seems to require more attention focused towards the motor control/postural task at hand, whereas complex secondary tasks may lead to increased postural sway compared to younger adults. The specific attention allocation patterns inherent in older adults during sensorimotor/cognitive task processing are based on the complex morphological and functional effects of aging in humans $[18,19]$. A common phenomenon related to aging and dualtasking is the prioritized division of attention and concurrent processing in specific task situations. As an example, older adults more often tend to stop walking when initiating a conversation with a walking companion [3]. Alternatively, often there is a shifting towards more of a motor prioritization that becomes more prominent in demanding postural perturbation tasks and balance threatening situations. Experimental results support the theory of a "posture-first" strategy when facing balance threats, which describes a focus of attention on the postural task in order to prevent falls in older adults, specifically those who are prone to falls [20]. Experiments exposing older adults to postural balance or gait stability threats, like heavy sway, obstacles, or sudden change of surface rigidity, showed a significant prioritization of postural control over cognitive processing either independent of aging or specifically in older adults [21, 22]. Modification of prioritization in specific postural situations can be seen as a plasticity mechanism [3] that is observable in healthy aging and patients suffering from sensory impairments [23].

Results from a number of studies highlight the significance of aging-related degeneration in central and peripheral sensorimotor processing and its effects on postural control $[1,2,24-28]$. Deterioration of the sensory system therefore affects postural control and in turn interacts with attention requirements and modified postural strategies in older adults, specifically in dual-task situations. Younger adults are able to adapt and to compensate for changes of sensory conditions [29]. However, it is also possible to modify and to improve sensory detection and processing, which may assist balance performance in older individuals and patients with neuropathies. A promising approach to achieve augmentation and better function of the somatosensory system is the utilization of interventions based on stochastic resonance (SR). SR is a phenomenon associated with induction of noise into a nonlinear system that is applicable to natural and manmade systems [30]. The term describes the enhancement of neural information transmission and weak stimuli detection when optimal noise is added to the system. The positive impact of SR on system functioning has been observed in a variety of sensory entities and over a wide range of tasks. Hence, tactile receptors and associated touch perception exhibit the beneficial features of SR enhancement. For instance, Well et al. showed that random vibration enhances the detection of weak touch, which has been observed for the foot soles as well [31]. If detection and processing of information can be enhanced via SR, this has significant implications for control of human motion and specifically for human postural control; hence, several studies have investigated the effects of tactile vibration to elicit SR effects on postural and gait performance [7, 32-37]. Considering the potential to enhance peripheral sensory detection and information transmission, it may be possible that a SRbased intervention may have effects on performance dualtask performance. This augmentation of feedback emerging from the foot soles could improve postural control efficiency, which would be associated with less cognitive demand, as potentially expressed either in better cognitive or postural performance in concurrent tasks. An improvement of postural performance in a dual-task has been observed in younger adults [35] but has not yet been investigated for older adults. Such investigations are important since older adults may benefit the most from a potentially balance-enhancing intervention; additionally, the effects of SR may be greater for older adults and those with lower baseline performance [38] or patients suffering from neuropathies [34].

It is still unclear to what extent this improvement of sensory afferent functioning might assist older individuals in performing postural tasks when additional cognitive processing load is added. To our knowledge, no study yet has methodologically investigated whether augmentation of somatosensory feedback does improve dual-task performance in older individuals or whether this intervention could lead to modifications of postural strategies in dual-task conditions.

We designed a study to investigate associations between postural performance and peripheral sensitivity and to investigate effects of aging and SR on healthy adults' postural control and performance. We hypothesized that there would be correlations between sensitivity and postural outcomes and that older adults and younger adults' postural outcomes would differ significantly. We further hypothesized that an SR intervention would affect participants' postural characteristics. 
TABLE 1: Anthropometric characteristics of younger and older participants and sensory threshold expressed as fraction of potential maximum amplitude output of the vibrotactile device.

\begin{tabular}{|c|c|c|c|c|c|c|c|}
\hline & $N$ & Gender & Height & Weight & Age & Foot length & $\%$ of vibration \\
\hline Younger & 10 & $\begin{array}{c}\mathrm{f} 5 \\
\mathrm{~m} 5\end{array}$ & $165.6 \pm 9.2$ & $148.3 \pm 27.2$ & $25.1 \pm 2.3$ & $25.4 \pm 2.3$ & $2.1 \pm 0.6$ \\
\hline Older & 10 & $\begin{array}{c}\mathrm{f} 8 \\
\mathrm{~m} 2\end{array}$ & $165.6 \pm 10.6$ & $151.1 \pm 35.2$ & $78.6 \pm 5.4$ & $25.4 \pm 1.6$ & $23.2 \pm 21.8$ \\
\hline
\end{tabular}

\section{Methods}

This study was conducted according to University of Houston policies concerning the protection of participants in human research. The protocol was approved by the University of Houston Committee for the Protection of Human Subjects (CPHS). All participants in the study provided informed written consent before participation.

2.1. Participants. Two groups of participants were recruited for this study, one healthy younger control group and one older experimental group (see Table 1).

Participants were included in the study if they were between the ages of either $20-35$ years or $70-85$ years. Physical health and cognitive function were initially evaluated based on a modified version of the Physical Activity Readiness Questionnaire and Mini Mental State Exam [39]. Participants were only included if they scored a minimum of 27 on the MMSE and did not report any significant impairments that could put them at risk during the experiment or may affect results. Only those with a BMI below 30 were included in the study. Additionally, participants were only included if they did (at the time of study participation) not use any medication that could interfere with their balance performance. An initial sensory detection test was administered to ensure that older adults displayed an increased tactile threshold at the foot sole, according to criteria described before [7]. Only those individuals who exhibited lower tactile sensitivity were included in the study. Foot length was measured as a requirement for the analysis of limits of stability (time-to-boundary). Demographics of the recruited participant groups are summarized in Table 1.

2.2. Equipment. A custom-made silicone insole was built (Hardness shore 50a) to integrate vibrotactile stimulators (C2; Engineering Acoustics, FL) that have been used in earlier SR studies $[7,34,38,40]$. The stimulators were integrated in the insole under the heel, the 1st and 5th metatarsalphalangeal joint region. The stimulators are magnet motor devices (diameter $30.5 \mathrm{~mm}$, height $7.9 \mathrm{~mm}$, and maximum displacement amplitude of about $0.635 \mathrm{~mm}$ ) that are connected to a control box including amplifiers and the power supply. In our study, the control box was connected to a PC via a USB cable. A computer-generated white-noise vibration signal band-limited from $1 \mathrm{~Hz}$ to about $500 \mathrm{~Hz}$ was used as the main mechanoreceptor stimulus. Customized software allowed the modulation of vibration amplitude to adapt it to individually required levels (Figure 1).
To pose a dual-task to participants, custom-made software was used to present verbal cues to the participants during each trial of the experiment via a headset. Verbal responses of the participants were recorded via the headset's microphone, whereas the software used speech recognition to compute both response latency and response accuracy (right/wrong answer).

2.2.1. Center-of-Pressure Data Collection. Center-of-pressure data was assessed using a force plate system (NeuroCom EquiTest, NeuroCom Intl., Clackamas, OR). Force plate data was collected at $100 \mathrm{~Hz}$ and processed via software on a connected computer (NeuroCom software version 8.0, NeuroCom Intl., Clackamas, OR).

2.3. Procedures. An initial test was conducted to determine if older participants were exhibiting different sensitivity levels related to mechanoreceptors of the foot sole. The testing was based on Semmes-Weinstein filament stimulation according to procedures described elsewhere [7].

After initial testing, participants were accustomed to the vibrating soles, which were adjusted to each participant's shoe size (several silicone strips in the mid-foot section could be added or removed to adjust size). After it was confirmed that the sole fitted well and all stimulators were in place, an initial vibration threshold test was performed. A stimulus intensity level of $90 \%$ of perception threshold $(100 \%)$ has been shown to be effective in SR stimulation experiments, so each participant's threshold was evaluated based on a method of levels [41], to gradually achieve an estimate of each individual's sensory threshold (ST).

In the following experimental trials, participants stood on the force plate for six $20 \mathrm{~s}$ trials, with $30 \mathrm{~s}$ breaks between each trial (and a two-minute break after three trials). They were instructed to stand quietly during each trial. Vibration conditions were randomized, so that there were three trials including vibration and three without. Due to the vibration amplitude set at $90 \%$, participants were not aware of the current vibration condition.

During each $20 \mathrm{~s}$ trial, participants were presented with a series of words via headphones (first word was presented at beginning of each trial, each subsequent word was presented in intervals of 4 seconds). The sequence of words was randomized by the software prior to each trial. Participants were asked to remember and then verbally repeat each word that was presented before the current one (1-back task). They were also asked to try to respond quickly and to speak clearly. Words consisted of the International Radiotelephony Spelling 


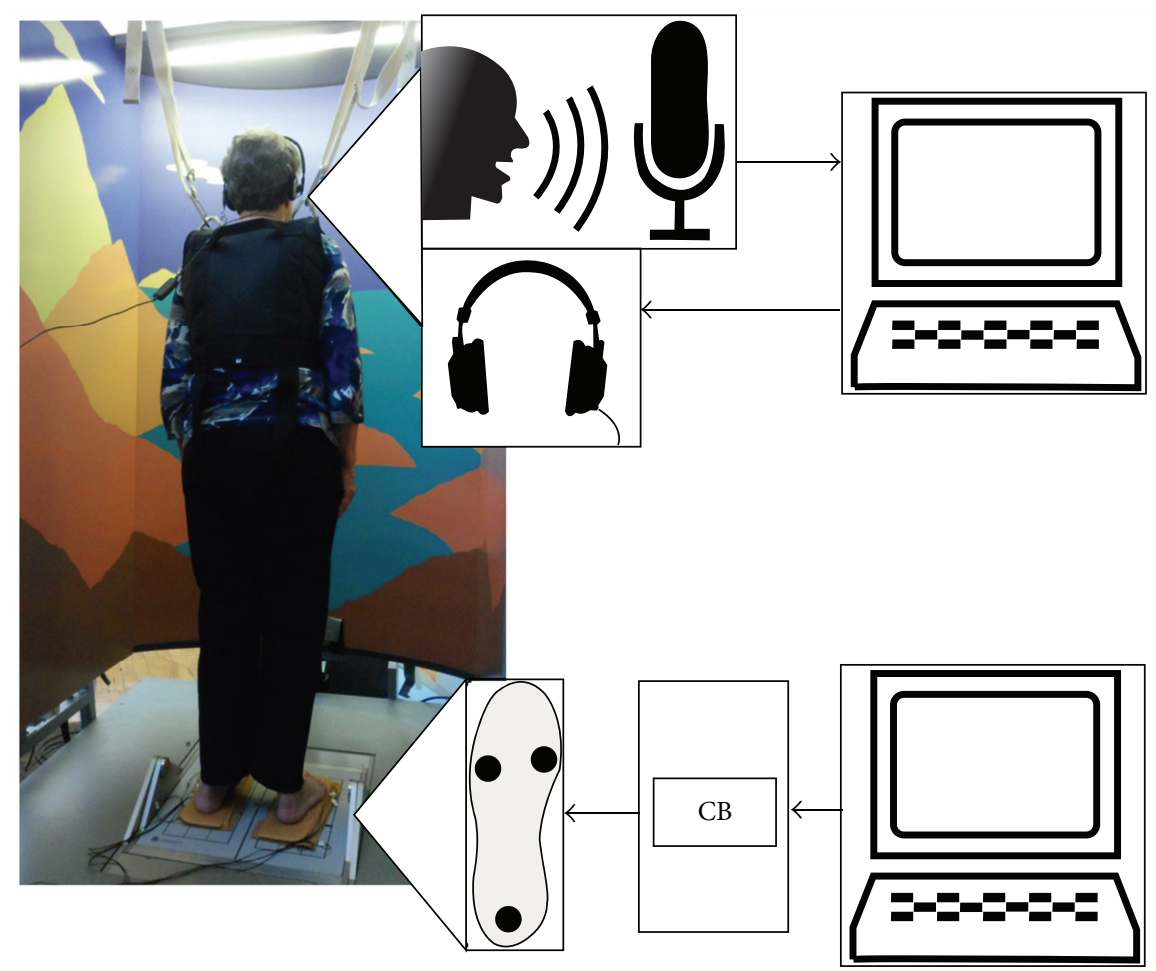

Figure 1: Schematic overview of the experimental setup. Participants received verbal cues to memorize and to recall (1-back task). Custommade software was used to provide cues and to analyze responses. Vibration was provided via tactor devices embedded in a silicone rubber sole. The soles were connected to a control box (CB) containing the power supply. The control box received commands from custom-made software on a connected computer. Not pictured: A trigger signal from the NeuroCom system was used to initiate stimulus presentation and data collection for the cognitive task.

Alphabet, whereas only polysyllabic items were included (24 different items). Initially, participants performed three trials (of $20 \mathrm{~s}$ each) of the task in standing position without force plate data collection. These training trials were performed to minimize adaptation to the cognitive task within the experimental trials.

2.4. Data Reduction. All outcome measures were computed using customized MATLAB (MATLAB 2012b, The MathWorks, Inc., Natick, MA) scripts and NeuroCom 8.0 software (NeuroCom, Clackamas, OR). Cognitive performance (error rate and reaction time) were assessed via customized software. Outcome measures were averaged for each subject over one block of trials (vibration on or vibration off).

2.4.1. Integrated Time-to-Boundary. Time-to-boundary (TTB) values were generated based on force plate data (measured at $100 \mathrm{~Hz}$ ). Velocity of the COP in anterior-posterior direction was first calculated based on earlier work [42]. Stability boundaries in the anterior-posterior direction were estimated based on the anterior-posterior limits of a rectangle involving the foot support base and initial foot length measurements. TTB was computed using the formula

$$
\mathrm{TTB}=\frac{d}{v}
$$

whereas $d$ is the distance to boundary $(d)$, estimated as the distance between the instantaneous COM location and the defined stability limits (boundary) in either given anteriorposterior direction at any moment and $v$ is velocity. An integrated area of TTB (iTTB) below a $10 \mathrm{~s}$ threshold was then computed for each trial to estimate general stability [42].

2.4.2. Root Mean Square (RMSAP) of COP. RMSAP as a measure of the magnitude of varying quantity was calculated from COP in anterior-posterior plane over the course of each trial of 20 seconds.

2.4.3. Approximate Entropy (ApEn). ApEn is a nonlinear measure that provides information about the regularity of a time series and has been applied to COP data in a number of different postural studies [43-46]. ApEn calculation was based on computations found elsewhere [47]. ApEn measures were generated using a customized MATLAB code and anterior-posterior COP displacement data for each trial. The data was processed with the following settings in the MATLAB analysis: A series length of 2 ( $m=2$ data points), an error tolerance window of 0.2 times the standard deviation of the respective time series $(r=0.2)$, and a lag value of 10 [48]. A single ApEn value for each trial was generated, which was then used for further statistical analysis and for 
surrogate analysis (as a necessary precursor to nonlinear/ ApEn analysis).

2.4.4. Equilibrium Score (ES). ES is a measure of postural stability based on hypothetical limits of stability. The formula to calculate ES is

$$
\mathrm{ES}=\frac{12.5-\left[\theta_{\max }-\theta_{\min }\right]}{12.5} * 100,
$$

where $\theta$ are sway angles and 12.5 is the estimated limit of sway (in degrees) for postural control [49]. The score ranges from 0 (a fall) to 100 (no sway).

2.4.5. Anterior-Posterior and Mediolateral Path Length (APPlength and MLPlength). The summation of all COP displacements over the course of each individual trial was calculated and is expressed through APPlength and MLPlength.

2.4.6. Anterior-Posterior and Mediolateral Maximal COP Excursion $\left(\mathrm{COPmax}_{A}\right.$ and COPmax $\mathrm{CO}_{P}$ ). The maximal excursion of the COP within each individual trial in both anterior and posterior direction was assessed as an indicator of instability; additionally, the combined maximal excursion in both directions was assessed.

2.4.7. Strategy Score (SS). SS evaluates movements around the upper body and hips and the lower body (ankles) that are generated for maintenance of postural stability.

The score is based on the formula

$$
1-\frac{\mathrm{SH}_{\max }-\mathrm{SH}_{\min }}{25} * 100 \text {, }
$$

where $\mathrm{SH}_{\max }$ and $\mathrm{SH}_{\text {min }}$ are the shear forces exerted to the force platform. A score of about 100 indicates a strategy based solely on an ankle strategy, and 0 would represent a strategy solely based on hip movements.

2.4.8. N-Back Cognitive Task: Response Time. For evaluation of cognitive performance in the experiment, participants' responses in each trial were analyzed. Data was collected using custom-made software that provided timed presentation of words. The software used Windows-based speech recognition to record both reaction time and correctness of responses during each trial. Correctness was evaluated by the software and defined by the participant correctly verbalizing the earlier (memorized) word right after the presentation of the currently presented one. Correctness was also evaluated by the investigators, who checked each response and noted incorrect responses in an Excel file during each trial. Responses that were incorrect but were corrected immediately by the participants were omitted from the data analysis. The main outcome of the cognitive portion of the experiment was a response time measure (timed at end of response), with response times averaged over all four responses of each trial.

2.5. Data Analysis. Statistical analyses of outcome measures were performed using SPSS v. 20 (IBM Corp., Somers, NY). Data are presented as group mean values \pm standard deviations (SD). Pearson product-moment correlation coefficients (Pearson's $r$ ) were computed to investigate associations between tactile sensitivity and postural measures. Mixedmodel ANOVA was used to investigate group differences and effects of SR. There was one between-groups factor (age) and one within-group factor (vibration). Analysis was conducted to investigate main effects (vibration and age) and potential interactions (age by vibration). Prior to computation of ANOVA statistics, data were analyzed to evaluate whether all required assumptions (for mixed ANOVA analysis) were fulfilled. Nonnormal distribution of data (as evaluated using Shapiro-Wilk tests with $\alpha$-levels set at 0.05 ) warranted the use of alternative, nonparametric statistical analysis. MannWhitney $U$ tests were used for comparisons of pairs of independent samples in this case. Bonferroni adjustment was used to account for multiple comparisons. Significance of statistical comparisons was set at $\alpha<.05$ level.

\section{Results}

Statistical analysis of the initial vibration threshold test revealed that the required vibration amplitude (to achieve $90 \%$ of the individual threshold) was significantly larger for the older group than for the younger group, $t(9.012), p=$ 0.013 .

A Pearson product-moment correlation coefficient was computed to assess the relationship between the tactile sensitivity as measured in the beginning of the experiment and different outcomes assessed during dual-tasking. There were several moderate to strong relationships, such as between sensory threshold (ST) and SS $(r=-.59, n=20, p=0.006)$, ST and iTTB $(r=.45, n=20, p=0.047)$, ST and COPmax A $_{\mathrm{A}}$ $(r=.54, n=20, p=0.015)$, ST and RMSAP $(r=.57, n=20$, $p=0.01)$, ST and APPlength $(r=.49, n=20, p=0.042)$, and ST and MLPlength $(r=.56, n=20, p=0.008)$.

Results from the dual-task experiment are summarized in Table 2. Older and younger participants differed regarding several outcome measures during dual-tasking, that is, $\operatorname{COPmax}_{\mathrm{A}}, F(1,18)=17.658, p=0.001$ (Figure 2), $\eta_{p}^{2}=.50$, $\mathrm{COPmax}_{\mathrm{P}}, F(1,18)=12.349, p=0.002, \eta_{p}^{2}=.41$ (Figure 3 ), RMSAP, $F(1,18)=5.956, p=0.025$ (Figure 4$), \eta_{p}^{2}=.25$, and MLPlength, $F(1,18)=5.473, p=0.031, \eta_{p}^{2}=.233$.

Nonparametric testing showed group differences for APPlength, which differed between older and younger adults both without vibration, $U=18, p=0.015, \eta_{p}^{2}=.29$, and with vibration, $U=15, p=0.007, \eta_{p}^{2}=.35$. Response time was also significantly different between groups without vibration, $U=1.5, p<0.001, \eta_{p}^{2}=.67$, and with vibration, $U=6, p=0.001, \eta_{p}^{2}=.55$ (Figure 5). There was no statistical significance for the main factor vibration, and there were no vibration-by-group interactions.

\section{Discussion}

The current experiment was designed to investigate the effects of aging and vibration on dual-task performance and control characteristics. We hypothesized that age and age-dependent 
TABLE 2: Means and standard deviations of postural performance, control, and cognitive response time of younger adults (YA) and older adults (OA) with and without vibration.

\begin{tabular}{|c|c|c|c|c|c|c|c|c|c|c|}
\hline & \multicolumn{2}{|c|}{ ITTB } & \multicolumn{2}{|c|}{ APPlength (in cm) } & \multicolumn{2}{|c|}{ MLPlength (in cm) } & \multicolumn{2}{|c|}{$\operatorname{COPmax}_{\mathrm{A}}($ in $\mathrm{cm})$} & \multicolumn{2}{|c|}{$\mathrm{COPmax}_{\mathrm{P}}($ in $\mathrm{cm})$} \\
\hline & YA & $\mathrm{OA}$ & YA & $\mathrm{OA}$ & YA & $\mathrm{OA}$ & YA & $\mathrm{OA}$ & YA & $\mathrm{OA}$ \\
\hline No vibration & $1.4 \pm 1.8$ & $2.6 \pm 4.2$ & $10.2 \pm 2.8$ & $17 \pm 7.9$ & $4.1 \pm 1.6$ & $6.2 \pm 2.1$ & $4.7 \pm 1.4$ & $7.1 \pm 1.0$ & $3.3 \pm 1.2$ & $5.2 \pm 1.3$ \\
\hline \multirow[t]{3}{*}{ Vibration } & $1.6 \pm 3.1$ & $2.4 \pm 3.6$ & $10.3 \pm 3.9$ & $16.9 \pm 6.8$ & $4.27 \pm 2.3$ & $1.6 \pm 3.1$ & $4.7 \pm 1.3$ & $6.8 \pm 1.1$ & $3.4 \pm 1.0$ & $5.0 \pm 1.1$ \\
\hline & \multicolumn{2}{|c|}{ RMSAP (in cm) } & \multicolumn{2}{|c|}{ Strategy score } & \multicolumn{2}{|c|}{ ES } & \multicolumn{2}{|c|}{ ApEn } & \multicolumn{2}{|c|}{ Response time } \\
\hline & YA & $\mathrm{OA}$ & YA & $\mathrm{OA}$ & YA & $\mathrm{OA}$ & YA & $\mathrm{OA}$ & YA & $\mathrm{OA}$ \\
\hline No vibration & $.42 \pm .2$ & $.58 \pm .1$ & $98.5 \pm .7$ & $98.0 \pm 1.0$ & $92.0 \pm 5.8$ & $92.0 \pm 2.3$ & $0.62 \pm .1$ & $0.68 \pm .2$ & $1.7 \pm .1$ & $2.1 \pm .3$ \\
\hline Vibration & $.41 \pm .2$ & $.59 \pm .2$ & $98.5 \pm .7$ & $98.0 \pm 1.1$ & $93.0 \pm 3.0$ & $92.6 \pm 1.4$ & $0.62 \pm .1$ & $0.69 \pm .1$ & $1.8 \pm .1$ & $2.2 \pm .3$ \\
\hline
\end{tabular}

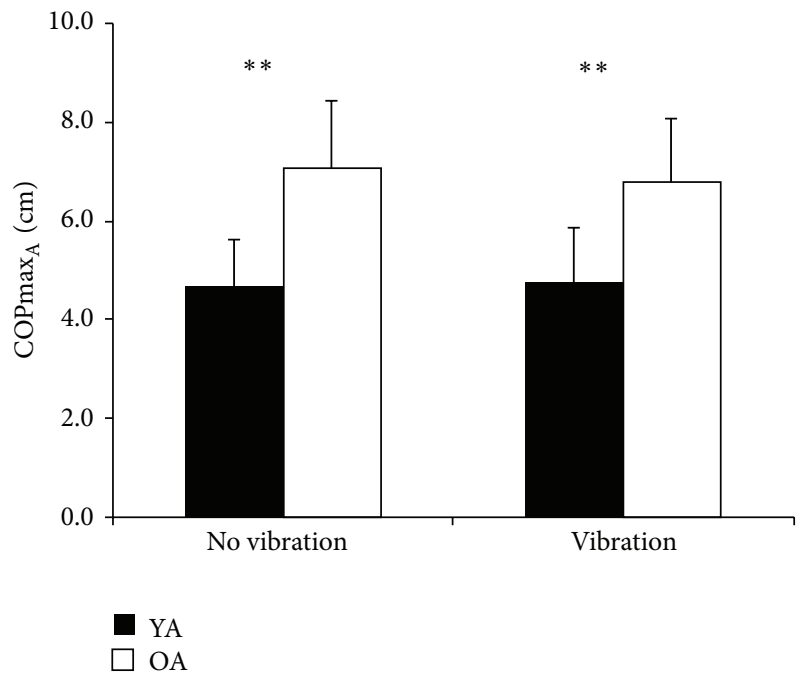

FIGURE 2: COPmax $\mathrm{A}_{\mathrm{A}}$ means and $\mathrm{SD}$ of younger age group (YA) and older age group $(\mathrm{OA})$ without vibration and with vibration. $* *=$ $p<0.01$.

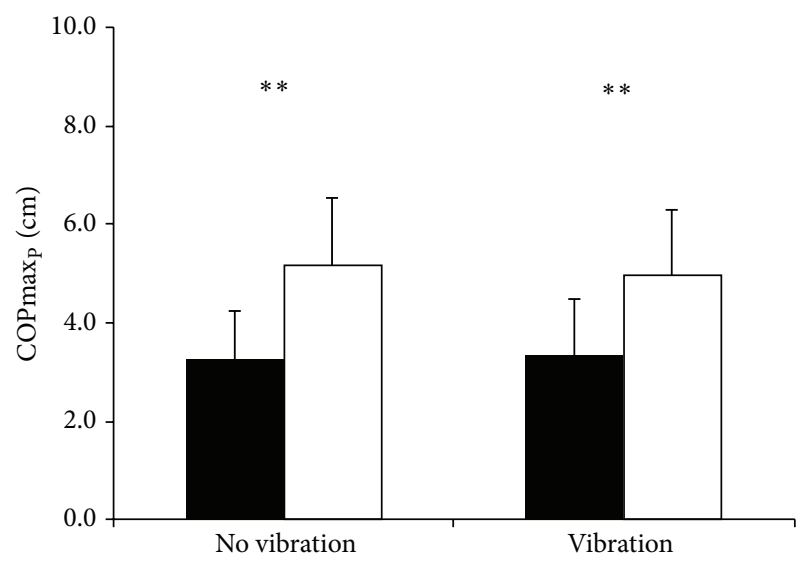

YA

$\square$ OA

FigURE 3: COPmax $\operatorname{P}_{\mathrm{P}}$ means and SD of younger age group (YA) and older age group $(\mathrm{OA})$ without vibration and with vibration. $* *=$ $p<0.01$.

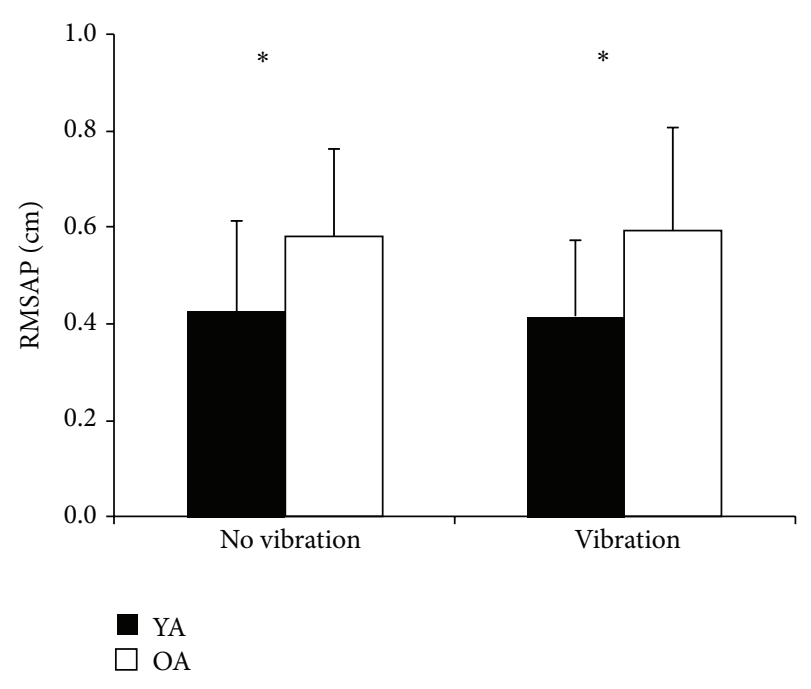

FIGURE 4: RMSAP means and SD of younger age group (YA) and older age group (OA) without vibration and with vibration. $*=p<$ 0.05 .

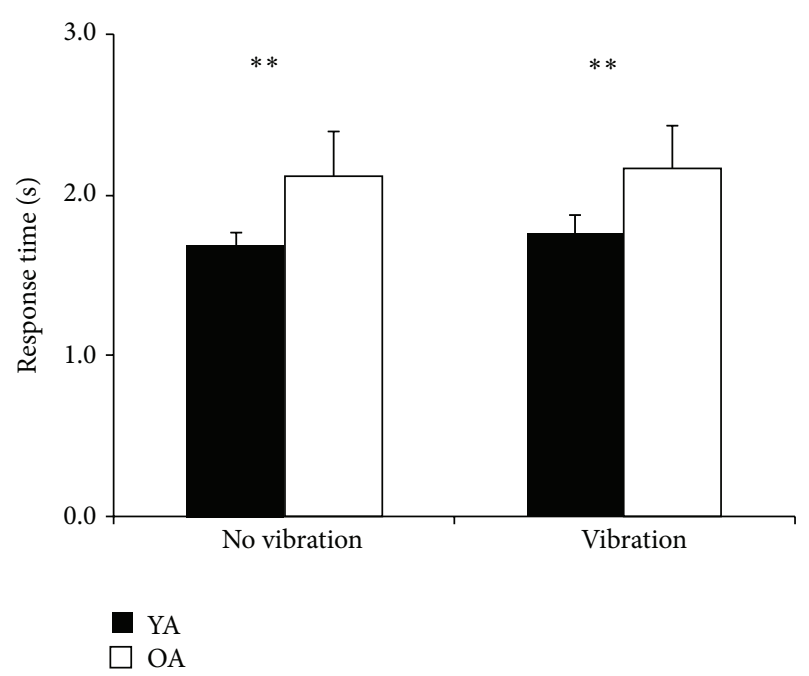

FIGURE 5: Response time means and SD of younger age group (YA) and older age group (OA) without vibration and with vibration. $* *=p<0.01$. 
loss of peripheral sensory function would be associated with performance and motor control in a dual-task situation and that outcomes would differ between conditions where vibration was either applied or not. We expected that the effects of aging on mental capacity and the sensorimotor decline observed with aging would affect outcomes related to one or both components of the dual-task.

Our hypothesis regarding associations between sensitivity of the foot sole and postural characteristics was confirmed, since several outcomes exhibited moderate to strong correlations with results from initial sensory testing. These findings confirm earlier results regarding the importance of tactile receptor feedback for postural tasks [50-53]. It has been postulated that deterioration of central integrative processes and peripheral sensitivity are contributors to postural control decline [54], and the current experiment provides evidence for this association in a dual-task situation. Additionally, the correlation observed between SS and sensitivity could be interpreted as an expression of sensorimotor adaptation that is required to maintain high levels of balance performance, as those participants who have less sensory feedback are known to adopt a strategy that includes more agonistantagonist coactivation on the lower leg for higher stiffness with more reliance on hip movements and less ankle movement. Deterioration of sensitivity at the foot soles may be a valuable predictor for balance issues; however, in the current experiment, older adults performed very well regarding the postural task despite increased sensory thresholds. This is remarkable, since older adults exhibiting high function regarding balance tasks or dual-tasking are also affected by sensory decline but potentially adapt to the deterioration by applying different movement strategies, for example, by using different multiple muscle activation patterns [55]. Alternatively, high-functioning older individuals may also have better control through postural reflexes, based on better function of the neuromuscular system in comparison to lowbalance performance individuals.

\subsection{Aging Effects on Balance Control and Performance. Older} and younger adults' balance performance differed regarding several but not all postural performance and control measures. This highlights the significance of assessing a number of different COP-based balance parameters, whereas such analyses can assist in the exploration of subtle changes of postural control due to aging, even in individuals whose balance performance is high.

APPlength/MLPlength, maximal COP excursion, and RMS of COP displacement are considered potential indicators of elevated postural instability, so the observed results regarding effects of aging on these outcomes could be interpreted as evidence for less stability in the older group when facing a dual-task challenge. However, considering the otherwise high performance levels of the specific group of older adults in this study, it is possible that path length and COP displacement increases in older adults were based on strategy modification, whereas participants allow for more sway to gather more sensory cues from the lower legs. This exploratory strategy may support the gathering of information [56] and ultimately would improve postural stability.
Results from iTTB and ES analysis did not reveal any group differences. The fact that older adults performed at similar levels to younger adults is unexpected but may be based on the specific task and the group of older adults that served as participants in the study. The $n$-back task performed concurrently in this experiment was designed to divert attention from postural control processes. It has been shown in younger adults that a fairly simple cognitive task (as is the 1-back task applied in our study) can actually lead to improved posture, associated with even less sway than when performing a single-task. The underlying idea is that an internal focus in an overlearned, mostly automatic, and selforganized task like quiet standing (e.g., based on the instruction "stand as quiet as possible") could interfere with the motor system [57-59]. The cognitive task in our experiment may have shifted the participants' focus towards an external cue, and automatic postural processes ensured maintenance of postural stability. This could explain why our results are contrary to earlier findings that suggested that older adults allowed about $40 \%$ increase of instability in favor of maintaining high performance in a concurrent cognitive task [8]. Doumas and colleagues administered a cognitive task that was more demanding than the task applied in the current study. It is likely that increased difficulty of the cognitive task would have shown pronounced age differences, based on increased resource competition. Additionally, the recall task that was applied in the current study differs from some of the tasks used in earlier studies, specifically regarding existing input-output modality pairings. In the current study, a verbalvocal task was used, which is considered a "compatible" pairing, and which has been shown to be less demanding than dual-tasks including "incompatible" modality pairings [60, 61]. Hence, these tasks require less cognitive effort, which may then be reflected in the postural performance component of an experiment. This highlights the need to consider aspects of modality compatibility in the design of future dual-task studies.

Participants in both groups were able to maintain overall high levels of stability, as evidenced by ES in the range of 90-95, and iTTB values indicating that COP-velocity and overall excursion towards the limits of stability were kept low.

However, we observed a potential trade-off in the cognitive domain. The response times in the $n$-back task administered in this experiment were different between older and younger adults. Older adults required more time to respond in the simple 1-back test compared to younger adults. It is possible that this observation indicates a trade-off between posture and cognitive processing, whereas longer response times were necessary to maintain high levels of postural performance. Alternatively, the trade-off may be a reflection of general aging processes between cognitive features, whereas older adults maintain accuracy of their responses (as observed in our study) while allowing for greater response times. This phenomenon has been observed before whereas, in certain tasks, reaction times are greater in older adults, but response accuracy is the same in comparison to younger adults [62]. This finding was accompanied by higher prefrontal cortex activity in older adults, potentially 
as a countermeasure to aging processes in the cognitive system [63]. It is possible that this increased cortical activity could make a difference when postural tasks (the primary task) become more difficult, for example, when the visual surrounding or the support surface is sway-referenced. Those tasks would require more conscious control of posture, and aging effects become more pronounced, as has been shown with more demanding secondary tasks [62]. The current results indicate that the older group accepted a trade-off regarding response time in favor of accuracy, a phenomenon that can occur when investigating both measures of accuracy and reaction time [64]. An alternative explanation is that the longer response latency in older adults could stem from prioritization differences due to aging. Participants were given the instruction to respond as quickly as possible, while standing as quiet as possible. Potentially, a higher prioritization was given to the postural task, affecting response times in this group. It is known that older adults have the ability to reallocate cognitive resources according to either instructions or due to strategic decisions, for example, postural stability over cognitive performance $[15,65]$. There were no wrong responses in either of the two groups, but it is possible that this would have changed if participants would have focused more on response time than on accuracy. Alternatively, a more demanding 2back test would have probably caused incorrect responses [62].

In contrast to our initial hypothesis, measures of postural control were not different between younger and older adults, as indicated by similar ApEn and strategy scores. This means that older and younger adults mainly used the same strategic approach to perform the dual-task. Concurrent processing of a cognitive task did not affect older adults in a manner that required them to change the strategy of attention sharing in comparison to younger adults. There arguably was no need for the older group to change the postural strategy compared to younger adults, as would have been evidenced by differences in strategy scores. For more demanding postural/cognitive tasks, more of a top-down strategy, including increased stiffness of the lower-leg musculature [66] and less ankle/more hip rotation, is expected, which is more pronounced in older adults. However, considering the nature of the experimental task in the current study, the need to adopt a top-down approach to postural control was not required.

This idea was supported by results from ApEn analysis. In the current task, temporal dynamics of COP variability were unaffected by age. ApEn seems to be dependent on amount of attention invested in postural control or a secondary task $[43,44]$. It can be concluded that the group of older adults included in this study did not adjust postural control to accommodate the requirements posed by the secondary cognitive task, as would have been indicated by changes of our entropy measure. Although ApEn has been shown to detect effects of the addition of a secondary task, even when initial postural sway was minimal [43], it may not be possible to detect age differences when highly functioning older adults are recruited.
4.2. Effects of Subthreshold Vibration. We had hypothesized that subthreshold vibration would alter postural performance, control characteristics, or cognitive performance, specifically in the older group. Our initial hypotheses concerning potential effects of subthreshold vibration on dualtasking, specifically in the elderly, were not confirmed.

Aging requires the allocation of more mental capacity or cognitive resources directed towards postural control or gait $[3,10,12]$. The enhancement of sensory feedback, especially about small excursions of the COP, could have effects on postural stability. This was not the case in the current experiment. The lack of any effects of vibration on either postural or cognitive measures indicates that the subtle enhancement of sensory feedback was not sufficient to affect outcomes. However, the overall high performance levels of the older adults group indicate that there was little necessity for improvement since performance mainly did not differ from the arguably near-optimal performance in the younger group (without vibration). It is unclear if the intervention used in this study could have positive effects if performance levels were lower, for example, in patient populations and recurrent fallers, specifically since SR effects are more pronounced when baseline levels are lower, which has been shown regarding the observed decreases of variability through SR in walking [38]. Additionally, it would be valuable to investigate if the intervention does have an effect in those individuals that suffer from mild or more severe cognitive impairments (and on their cognitive or postural performance).

The connection between postural stability, dual-tasking, and cognitive impairments has been previously identified. As has been concluded from findings in a recent study including a large number of older adults $(n=717)$, it is possible that dual-tasking performance correlates more with fall risk among individuals that suffer from pathological conditions than those who are healthy [66]. The older group recruited for the current study consisted of high-functioning individuals, who live mostly independently and who did not have any cognitive impairment. Earlier research has shown an age-dependent increase in the correlation between cognitive/intellectual abilities and sensorimotor function [19], with an increasingly negative age-dependent correlation between sensorimotor fluctuation and cognitive abilities [67]. The group in our study exhibited higher sensory thresholds but were very similar to the younger group concerning performance and postural control measures. Therefore, the high levels of cognitive function and sensorimotor function retained by this recruited group allowed for performance and control that was similar regarding some performance outcomes, with vibration having no effects on outcomes in either group.

The participant group in this study probably affected results, and considering the exclusion and inclusion criteria that we established, only healthy older adults with high levels of function were recruited. Although those individuals may exhibit higher sensory thresholds and slight decline regarding postural performance compared to younger individuals, differences were relatively small. Further research could aim at investigating the effects of the presented intervention in 
individuals suffering from mild or more severe cognitive impairment, which could interfere with postural control in dual-tasks. It would be valuable to evaluate whether the intervention can affect performance in those individuals, compared to the current results in healthy older adults.

\section{Conclusions}

Results from the current study indicate specific correlations between tactile sensitivity and postural performance and control in a simple dual-task. The evaluation of tactile sensitivity in older adults for the purpose of prediction of fall risk or postural performance may not be adequate for otherwise high-functioning individuals. Healthy aging affects several postural outcomes in dual-tasking, but the nature of the dual-task and associated modality compatibility may affect results. This has implications for future study designs and the interpretability of results regarding a translation to real-life situations.

A tactile SR intervention may not improve performance if the task is simple, or when participants exhibit high-baseline performance. The application of the technology in a clinical setting may therefore benefit from extensive initial testing, whereas SR-based interventions may be only valuable for certain individuals. Future research should investigate effects of SR in more demanding tasks, in a number of dual-tasks using different sets of modality-mappings, and in individuals suffering from severe sensorimotor impairments.

\section{Competing Interests}

The authors declare that they have no competing interests.

\section{References}

[1] D. L. Sturnieks, R. St George, and S. R. Lord, "Balance disorders in the elderly," Clinical Neurophysiology, vol. 38, no. 6, pp. 467478, 2008.

[2] F. B. Horak, C. L. Shupert, and A. Mirka, "Components of postural dyscontrol in the elderly: a review," Neurobiology of Aging, vol. 10, no. 6, pp. 727-738, 1989.

[3] M. Lacour, L. Bernard-Demanze, and M. Dumitrescu, "Posture control, aging, and attention resources: models and postureanalysis methods," Neurophysiologie Clinique, vol. 38, no. 6, pp. 411-421, 2008.

[4] M. H. Woollacott and A. Shumway-Cook, "Changes in posture control across the life span-a systems approach," Physical Therapy, vol. 70, no. 12, pp. 799-807, 1990.

[5] J. J. Siracuse, D. D. Odell, S. P. Gondek et al., "Health care and socioeconomic impact of falls in the elderly," American Journal of Surgery, vol. 203, no. 3, pp. 335-338, 2012.

[6] L. Berger and L. Bernard-Demanze, "Age-related effects of a memorizing spatial task in the adults and elderly postural control," Gait and Posture, vol. 33, no. 2, pp. 300-302, 2011.

[7] M. Dettmer, A. Pourmoghaddam, B.-C. Lee, and C. S. Layne, "Effects of aging and tactile stochastic resonance on postural performance and postural control in a sensory conflict task," Somatosensory and Motor Research, vol. 32, no. 2, pp. 128-135, 2015.
[8] M. Doumas, C. Smolders, and R. T. Krampe, "Task prioritization in aging: effects of sensory information on concurrent posture and memory performance," Experimental Brain Research, vol. 187, no. 2, pp. 275-281, 2008.

[9] M. Doumas, M. A. Rapp, and R. T. Krampe, "Working memory and postural control: adult age differences in potential for improvement, task priority, and dual tasking," Journals of Gerontology B. Psychological Sciences and Social Sciences, vol. 64, no. 2, pp. 193-201, 2009.

[10] R. T. Krampe, M. A. Rapp, A. Bondar, and P. B. Baltes, "Allocation of cognitive resources during the simultaneous performance of cognitive and sensorimotor tasks," Nervenarzt, vol. 74, no. 3, pp. 211-218, 2003.

[11] A. P. Marsh and S. E. Geel, "The effect of age on the attentional demands of postural control," Gait and Posture, vol. 12, no. 2, pp. 105-113, 2000.

[12] Y. Lajoie, N. Teasdale, C. Bard, and M. Fleury, "Upright standing and gait: are there changes in attentional requirements related to normal aging?" Experimental Aging Research, vol. 22, no. 2, pp. 185-198, 1996.

[13] I. Melzer, N. Benjuya, and J. Kaplanski, "Age-related changes of postural control: effect of cognitive tasks," Gerontology, vol. 47, no. 4, pp. 189-194, 2001.

[14] I. Olivier, R. Cuisinier, M. Vaugoyeau, V. Nougier, and C. Assaiante, "Age-related differences in cognitive and postural dualtask performance," Gait and Posture, vol. 32, no. 4, pp. 494-499, 2010.

[15] K. Z. H. Li, R. T. Krampe, and A. Bondar, "An ecological approach to studying aging and dual-task performance," in Cognitive Limitations in Aging and Psychopathology, R. W. Engle, G. Sedek, U. von Hecker, and D. N. McIntosh, Eds., pp. 190-218, Cambridge University Press, New York, NY, USA, 2005.

[16] L. A. Brown, A. Shumway-Cook, and M. H. Woollacott, "Attentional demands and postural recovery: the effects of aging," The Journals of Gerontology_Series A, vol. 54, no. 4, pp. M165-M171, 1999.

[17] N. Teasdale and M. Simoneau, "Attentional demands for postural control: the effects of aging and sensory reintegration," Gait and Posture, vol. 14, no. 3, pp. 203-210, 2001.

[18] S.-C. Li and H. R. Dinse, "Aging of the brain, sensorimotor, and cognitive processes," Neuroscience and Biobehavioral Reviews, vol. 26, no. 7, pp. 729-732, 2002.

[19] K. Z. H. Li and U. Lindenberger, "Relations between aging sensory/sensorimotor and cognitive functions," Neuroscience and Biobehavioral Reviews, vol. 26, no. 7, pp. 777-783, 2002.

[20] A. Shumway-Cook and M. Woollacott, "Attentional demands and postural control: the effect of sensory context," Journals of Gerontology, Series A: Biological Sciences and Medical Sciences, vol. 55, no. 1, pp. M10-M16, 2000.

[21] F. Mersmann, S. Bohm, S. Bierbaum, R. Dietrich, and A. Arampatzis, "Young and old adults prioritize dynamic stability control following gait perturbations when performing a concurrent cognitive task," Gait and Posture, vol. 37, no. 3, pp. 373-377, 2013.

[22] A. Shumway-Cook, M. Woollacott, K. A. Kerns, and M. Baldwin, "The effects of two types of cognitive tasks on postural stability in older adults with and without a history of falls," Journals of Gerontology A. Biological Sciences and Medical Sciences, vol. 52, no. 4, pp. M232-M240, 1997.

[23] M. Lacour, "Restoration of vestibular function: basic aspects and practical advances for rehabilitation," Current Medical Research and Opinion, vol. 22, no. 9, pp. 1651-1659, 2006. 
[24] I. Amiridis, V. Hatzitaki, and F. Arabatzi, "Age-induced modifications of static postural control in humans," Neuroscience Letters, vol. 350, no. 3, pp. 137-140, 2003.

[25] D. Abrahamová and F. Hlavacka, "Age-related changes of human balance during quiet stance," Physiological Research/ Academia Scientiarum Bohemoslovaca, vol. 57, no. 6, pp. 957964, 2008.

[26] M. Bosek, B. Grzegorzewski, A. Kowalczyk, and I. Lubiński, "Degradation of postural control system as a consequence of Parkinson's disease and ageing," Neuroscience Letters, vol. 376, no. 3, pp. 215-220, 2005.

[27] R. D. Seidler, J. A. Bernard, T. B. Burutolu et al., "Motor control and aging: links to age-related brain structural, functional, and biochemical effects," Neuroscience and Biobehavioral Reviews, vol. 34, no. 5, pp. 721-733, 2010.

[28] S. W. Shaffer and A. L. Harrison, "Aging of the somatosensory system: a translational perspective," Physical Therapy, vol. 87, no. 2, pp. 193-207, 2007.

[29] M. Dettmer, A. Pourmoghaddam, D. P. O’Connor, and C. S. Layne, "Interaction of support surface stability and Achilles tendon vibration during a postural adaptation task," Human Movement Science, vol. 32, no. 1, pp. 214-227, 2013.

[30] F. Moss, L. M. Ward, and W. G. Sannita, "Stochastic resonance and sensory information processing: a tutorial and review of application," Clinical Neurophysiology, vol. 115, no. 2, pp. 267281, 2004

[31] C. Well, L. M. Ward, R. Chua, and J. Timothy Inglis, “Touch noise increases vibrotactile sensitivity in old and young," Psychological Science, vol. 16, no. 4, pp. 313-320, 2005.

[32] M. Costa, A. A. Priplata, L. A. Lipsitz et al., "Noise and poise: enhancement of postural complexity in the elderly with a stochastic-resonance-based therapy," Europhysics Letters, vol. 77, no. 6, Article ID 68008, 2007.

[33] J. J. Collins, A. A. Priplata, D. C. Gravelle, J. Niemi, J. Harry, and L. A. Lipsitz, "Noise-enhanced human sensorimotor function," IEEE Engineering in Medicine and Biology Magazine, vol. 22, no. 2, pp. 76-83, 2003.

[34] A. A. Priplata, B. L. Patritti, J. B. Niemi et al., "Noise-enhanced balance control in patients with diabetes and patients with stroke," Annals of Neurology, vol. 59, no. 1, pp. 4-12, 2006.

[35] E. A. Keshner, J. C. Slaboda, L. L. Day, and K. Darvish, "Visual conflict and cognitive load modify postural responses to vibrotactile noise," Journal of NeuroEngineering and Rehabilitation, vol. 11, article 6, 2014.

[36] J. M. Hijmans, J. H. B. Geertzen, W. Zijlstra, A. L. Hof, and K. Postema, "Effects of vibrating insoles on standing balance in diabetic neuropathy," Journal of Rehabilitation Research and Development, vol. 45, no. 9, pp. 1441-1450, 2008.

[37] L. A. Lipsitz, M. Lough, J. Niemi, T. Travison, H. Howlett, and B. Manor, "A shoe insole delivering subsensory vibratory noise improves balance and gait in healthy elderly people," Archives of Physical Medicine and Rehabilitation, vol. 96, no. 3, pp. 432-439, 2015.

[38] D. G. Stephen, B. J. Wilcox, J. B. Niemi, J. Franz, D. Casey Kerrigan, and S. E. D'Andrea, "Baseline-dependent effect of noiseenhanced insoles on gait variability in healthy elderly walkers," Gait and Posture, vol. 36, no. 3, pp. 537-540, 2012.

[39] M. F. Folstein, S. E. Folstein, and P. R. McHugh, "'Mini-mental state. A practical method for grading the cognitive state of patients for the clinician," Journal of Psychiatric Research, vol. 12, no. 3, pp. 189-198, 1975.
[40] A. M. Galica, H. G. Kang, A. A. Priplata et al., "Subsensory vibrations to the feet reduce gait variability in elderly fallers," Gait and Posture, vol. 30, no. 3, pp. 383-387, 2009.

[41] M. E. Shy, E. M. Frohman, Y. T. So et al., "Quantitative sensory testing: report of the therapeutics and technology assessment subcommittee of the American academy of neurology," Neurology, vol. 60, no. 6, pp. 898-904, 2003.

[42] R. A. Ozdemir, A. Pourmoghaddam, and W. H. Paloski, "Sensorimotor posture control in the blind: superior ankle proprioceptive acuity does not compensate for vision loss," Gait and Posture, vol. 38, no. 4, pp. 603-608, 2013.

[43] J. T. Cavanaugh, V. S. Mercer, and N. Stergiou, "Approximate entropy detects the effect of a secondary cognitive task on postural control in healthy young adults: a methodological report," Journal of NeuroEngineering and Rehabilitation, vol. 4, article 42, 2007.

[44] S. F. Donker, M. Roerdink, A. J. Greven, and P. J. Beek, "Regularity of center-of-pressure trajectories depends on the amount of attention invested in postural control," Experimental Brain Research, vol. 181, no. 1, pp. 1-11, 2007.

[45] S. L. Hong, B. Manor, and L. Li, "Stance and sensory feedback influence on postural dynamics," Neuroscience Letters, vol. 423, no. 2, pp. 104-108, 2007.

[46] C. K. Rhea, T. A. Silver, S. L. Hong et al., "Noise and complexity in human postural control: interpreting the different estimations of entropy," PLoS ONE, vol. 6, no. 3, Article ID e17696, 2011.

[47] H. Ocak, "Automatic detection of epileptic seizures in EEG using discrete wavelet transform and approximate entropy," Expert Systems with Applications, vol. 36, no. 2, pp. 2027-2036, 2009.

[48] J. T. Cavanaugh, K. M. Guskiewicz, C. Giuliani, S. Marshall, V. S. Mercer, and N. Stergiou, "Recovery of postural control after cerebral concussion: new insights using approximate entropy," Journal of Athletic Training, vol. 41, no. 3, pp. 305-313, 2006.

[49] H. Chaudhry, B. Bukiet, Z. Ji, and T. Findley, "Measurement of balance in computer posturography: comparison of methodsa brief review," Journal of Bodywork and Movement Therapies, vol. 15, no. 1, pp. 82-91, 2011.

[50] S. R. Lord, R. D. Clark, and I. W. Webster, "Postural stability and associated physiological factors in a population of aged persons," Journals of Gerontology, vol. 46, no. 3, pp. M69-76, 1991.

[51] B. E. Maki, S. D. Perry, R. G. Nome, and W. E. Mcllroy, "Effect of facilitation of sensation from plantar foot-surface boundaries on postural stabilization in young and older adults," Journals of Gerontology A. Biological Sciences and Medical Sciences, vol. 54, no. 6, pp. M281-M287, 1999.

[52] P. F. Meyer, L. I. E. Oddsson, and C. J. De Luca, "The role of plantar cutaneous sensation in unperturbed stance," Experimental Brain Research, vol. 156, no. 4, pp. 505-512, 2004.

[53] M. Patel, M. Magnusson, E. Kristinsdottir, and P.-A. Fransson, "The contribution of mechanoreceptive sensation on stability and adaptation in the young and elderly," European Journal of Applied Physiology, vol. 105, no. 2, pp. 167-173, 2009.

[54] E. Palluel, I. Olivier, and V. Nougier, "The lasting effects of spike insoles on postural control in the elderly," Behavioral Neuroscience, vol. 123, no. 5, pp. 1141-1147, 2009. 
[55] A. Pourmoghaddam, M. Dettmer, D. P. O'Connor, W. H. Paloski, and C. S. Layne, "Identification of changing lower limb neuromuscular activation in Parkinson's disease during treadmill gait with and without levodopa using a nonlinear analysis index," Parkinson's Disease, vol. 2015, Article ID 497825, 8 pages, 2015.

[56] M. A. Riley, R. Balasubramaniam, and M. T. Turvey, "Recurrence quantification analysis of postural fluctuations," Gait and Posture, vol. 9, no. 1, pp. 65-78, 1999.

[57] O. Huxhold, S.-C. Li, F. Schmiedek, and U. Lindenberger, "Dualtasking postural control: aging and the effects of cognitive demand in conjunction with focus of attention," Brain Research Bulletin, vol. 69, no. 3, pp. 294-305, 2006.

[58] J. Verrel, M. Lövdén, M. Schellenbach, S. Schaefer, and U. Lindenberger, "Interacting effects of cognitive load and adult age on the regularity of whole-body motion during treadmill walking," Psychology and Aging, vol. 24, no. 1, pp. 75-81, 2009.

[59] R. C. O'Reilly, "Biologically based computational models of high-level cognition," Science, vol. 314, no. 5796, pp. 91-94, 2006.

[60] E. Hazeltine, E. Ruthruff, and R. W. Remington, "The role of input and output modality pairings in dual-task performance: evidence for content-dependent central interference," Cognitive Psychology, vol. 52, no. 4, pp. 291-345, 2006.

[61] C. Stelzel, E. H. Schumacher, T. Schubert, and M. D'Esposito, "The neural effect of stimulus-response modality compatibility on dual-task performance: an fMRI study," Psychological Research, vol. 70, no. 6, pp. 514-525, 2006.

[62] V. S. Mattay, F. Fera, A. Tessitore et al., "Neurophysiological correlates of age-related changes in working memory capacity," Neuroscience Letters, vol. 392, no. 1-2, pp. 32-37, 2006.

[63] C. L. Grady, "Cognitive neuroscience of aging," Annals of the New York Academy of Sciences, vol. 1124, pp. 127-144, 2008.

[64] A. Remaud, S. Boyas, G. A. Caron, and M. Bilodeau, "Attentional demands associated with postural control depend on task difficulty and visual condition," Journal of Motor Behavior, vol. 44, no. 5, pp. 329-340, 2012.

[65] M. Riediger, S.-C. Li, and U. Lindenberger, "Selection, optimization, and compensation as developmental mechanisms of adaptive resource allocation: review and preview," in Handbook of the Psychology of Aging, J. E. Birren, K. W. Schaie, R. P. Abeles, M. Gatz, and T. A. Salthouse, Eds., pp. 289-313, Elsevier, Burlington, Mass, USA, 6th edition, 2006.

[66] H. G. Kang, L. Quach, W. Li, and L. A. Lipsitz, "Stiffness control of balance during dual task and prospective falls in older adults: the MOBILIZE Boston Study," Gait and Posture, vol. 38, no. 4, pp. 757-763, 2013.

[67] S.-C. Li, U. Lindenberger, and S. Sikström, "Aging cognition: from neuromodulation to representation," Trends in Cognitive Sciences, vol. 5, no. 11, pp. 479-486, 2001. 


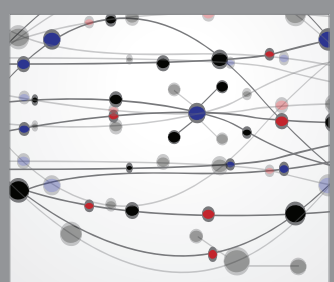

The Scientific World Journal
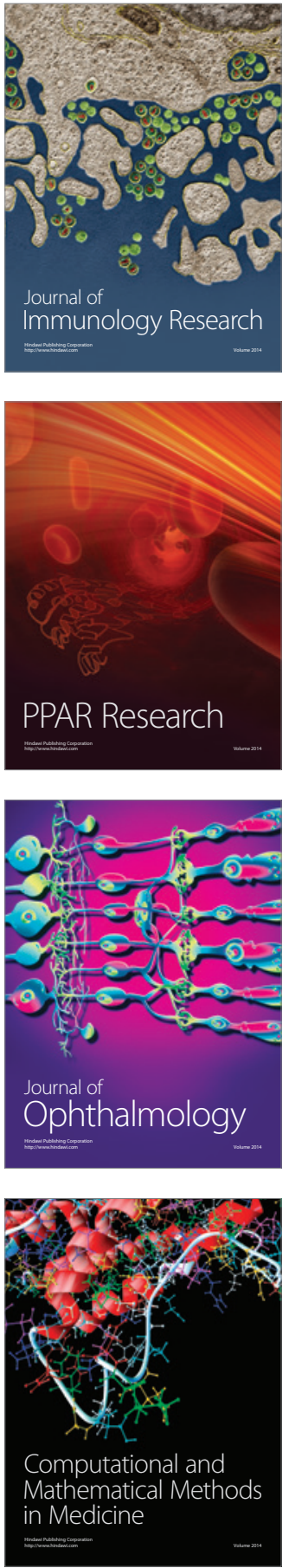

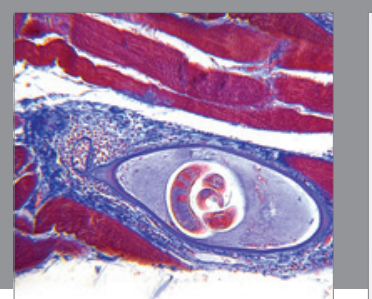

Gastroenterology Research and Practice

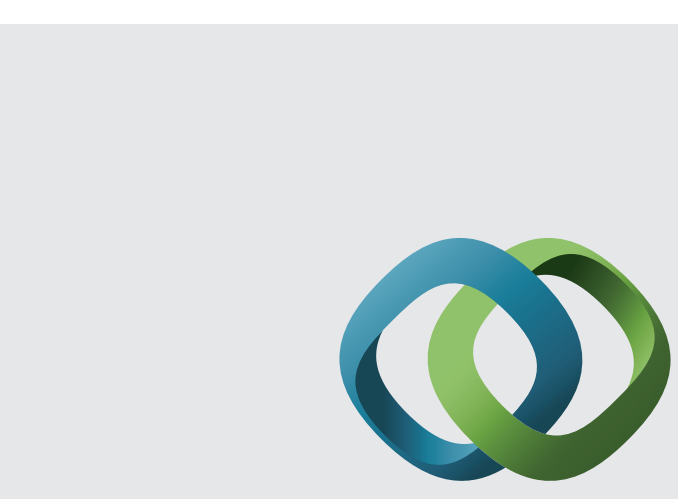

\section{Hindawi}

Submit your manuscripts at

http://www.hindawi.com
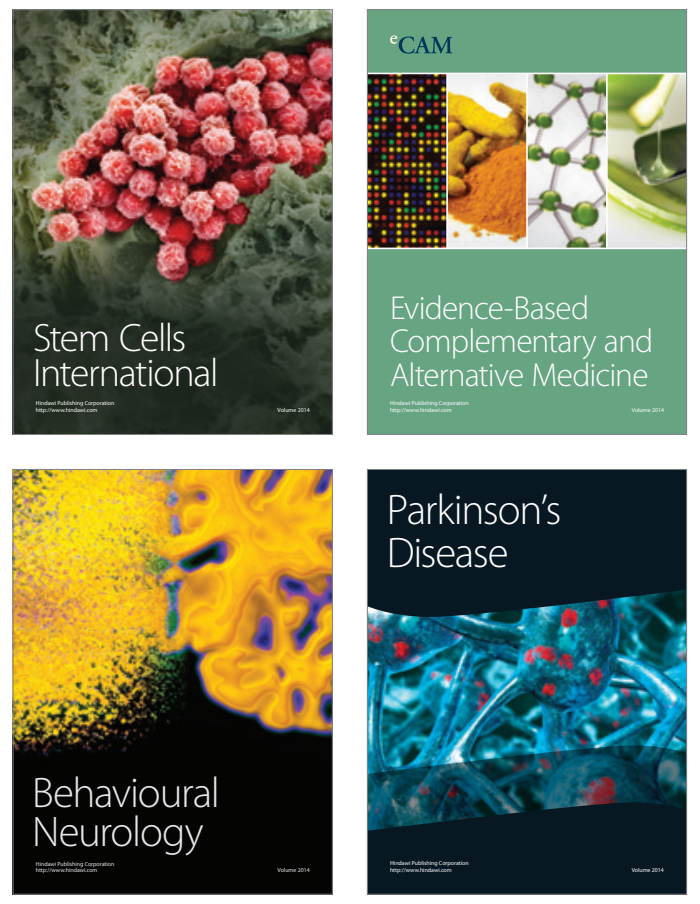
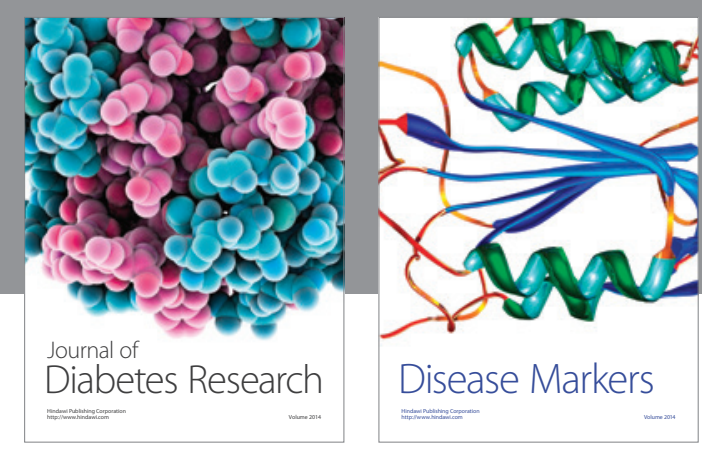

Disease Markers
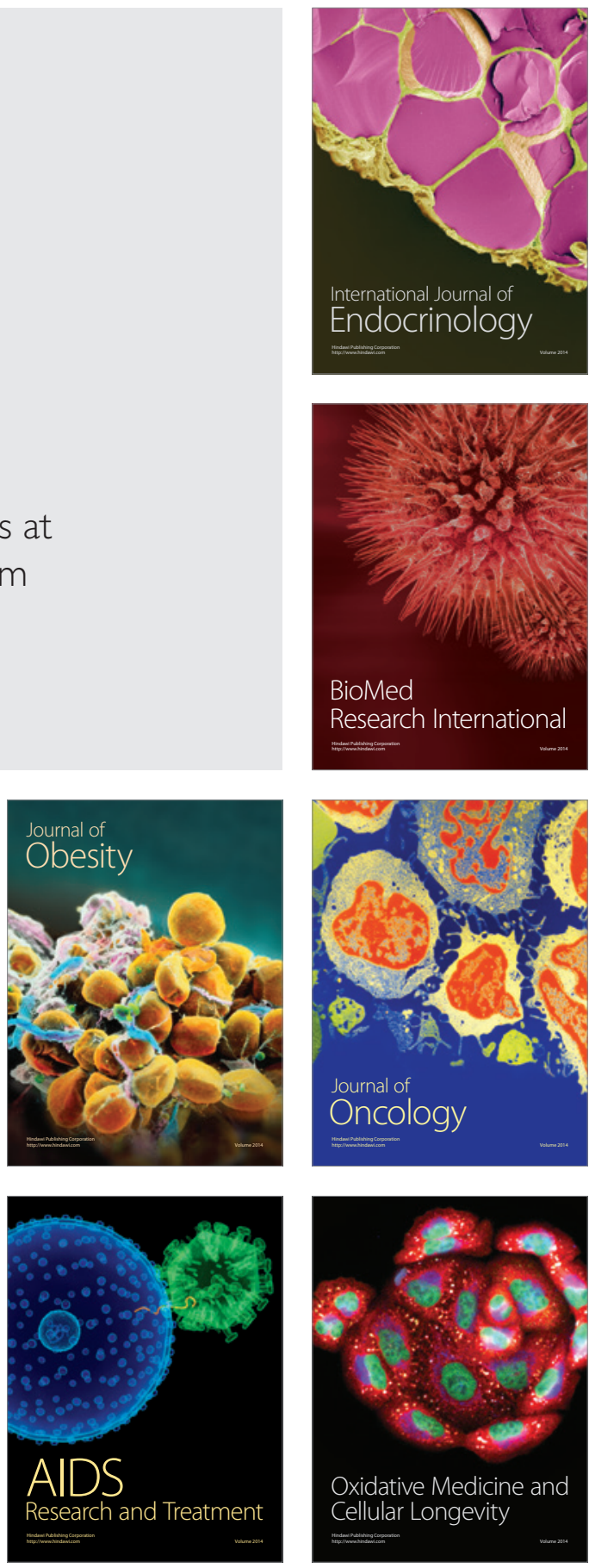